\title{
An unusual presentation of a digital schwannoma: case report
}

\author{
C. Liam Dwyer • Andrew J. Creager • John D. Lubahn
}

Published online: 7 January 2014

(C) American Association for Hand Surgery 2014

\section{Introduction}

Schwannomas are primary benign peripheral nerve sheath tumors. They are also known as neurilemmomas and represent less than $5 \%$ of all upper extremity tumors [1] and $2.8 \%$ of biopsied benign soft-tissue tumors of the hand and wrist [10]. Although most commonly found in the head and neck, schwannomas of the hand and wrist have been reported and reviewed [12]. Schwannomas of the upper extremity typically appear on the volar surface of the hand or arm. There have also been reports of unusual cases of schwannomas associated with macrodactyly [14], associated with trigger finger [16], located in the subungal region of the fingertip [8], located intraosseously [2, 11], and in the fingers of children [18, 19]. A search of the English language literature failed to reveal any reports describing the atypical presentation of a schwannoma originally diagnosed as a mucous cyst or located at the dorsal aspect of the distal interphalangeal (DIP) joint.

This report presents a case of a myxoid schwannoma mimicking the clinical findings of a typical mucous cyst. Based on the surgical findings after excision and subsequent pathologic studies, the patient was diagnosed as having a myxoid schwannoma.

\section{Case Report}

A 50-year-old female presented for evaluation of a solitary painless cystic appearing mass measuring approximately

C. L. Dwyer · A. J. Creager · J. D. Lubahn

UPMC Hamot, 201 State Street, Erie, PA 16550, USA

J. D. Lubahn $(\bowtie)$

Hand Microsurgery and Reconstructive Orthopedics, LLP,

300 State Street, Suite 205, Erie, PA 16507, USA

e-mail: jdlubahn@jdlubahn.com

$5 \mathrm{~mm}$ in diameter with very minimal surrounding erythema off of midline on the dorsal aspect of her left long finger and just proximal to the nail at the level of the DIP joint (Fig. 1). The patient first noticed the mass approximately 12 months prior to initial presentation. The soft and immobile lesion was nontender to palpation, and there was full motion of the DIP joint. There was normal sensation and capillary refill distal to the lesion. She denied any size fluctuation or associated symptoms of pain or dysesthesia. Radiographic examination demonstrated no obvious fracture, dislocation, signs of degenerative change, or cortical disruption (Fig. 2). Only a minimal amount of soft tissue swelling was visualized dorsal to the distal phalanx without any defined soft tissue mass or boney involvement identified. As the patient was dissatisfied with the cosmetic appearance of the lesion and the noted associated grooving of the nail plate, she elected to have the lesion removed.

The presentation and exam of the patient were consistent with that of a typical mucous cyst. Specifically, the asymptomatic lesion was located off of midline dorsally at the level of the DIP joint within the proximal nail fold and had associated nail ridging. Therefore, the patient underwent surgical excision through a dorsal $\mathrm{H}$-shaped incision at the DIP joint. Full thickness flaps were elevated to expose the mass with careful attention paid to protecting the extensor tendon, neurovascular bundles, and germinal matrix. Upon exposure of the mass, it was immediately evident that this lesion was not a mucous cyst. The subcutaneous atypical yellowish myxoid lesion was carefully excised (Fig. 3) using 3.5 power Loupe magnification along with a minimal amount of surrounding adipose tissue. No germinal stalk or associated neurologic structure was appreciated. As there was no identified osteophyte radiographically, and it was determined that the lesion was not a mucous cyst, boney debridement was deemed unnecessary, and the capsule was left intact. After irrigation, the skin incision was closed with nylon suture, and the digit was dressed and splinted in extension. 


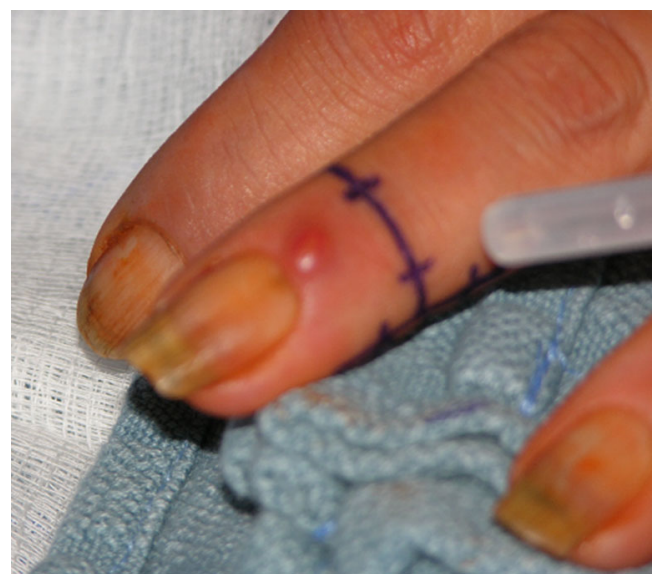

Fig. 1 Clinical photograph demonstrating the cystic appearing mass on the dorsal aspect of her left long finger at the level of the distal interphalangeal joint with an associated grooved nail plate

Histologic examination confirmed the presence of an encapsulated schwannoma with myxoid stroma, focal areas of arranged spindle cell proliferation (Antoni type A) and hypocellular (Antoni type B) areas (Fig. 4a and $b$ ). Along with this, the S-100 immunoreactivity of the spindle cells supported the Schwann cell origin of the lesion.

The diagnosis of myxoid schwannoma was established. Postoperatively, the patient maintained brisk capillary refill and unchanged sensation distal to the lesion. The patient is satisfied with her treatment and remains asymptomatic without any pain, neurologic symptoms,

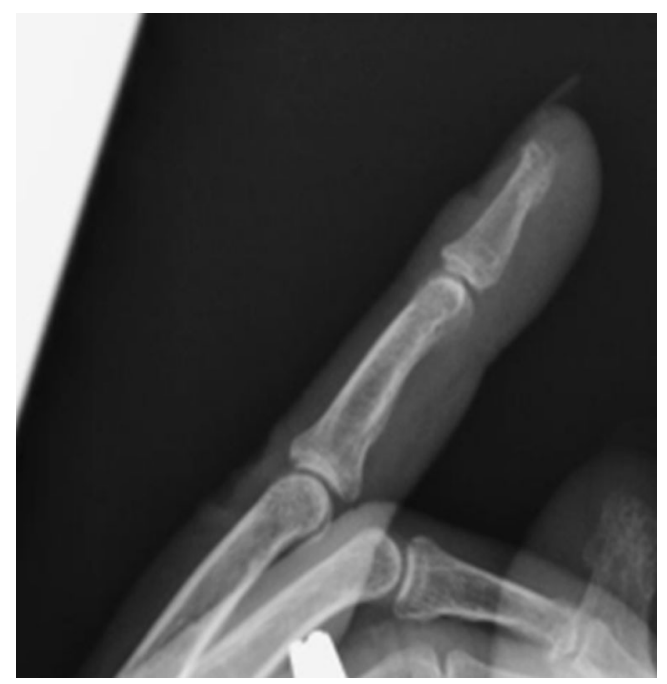

Fig. 2 Lateral radiograph of the left long finger demonstrating minimal swelling about the dorsal distal phalanx and mild degenerative changes, without signs of cortical disruption or boney lesion

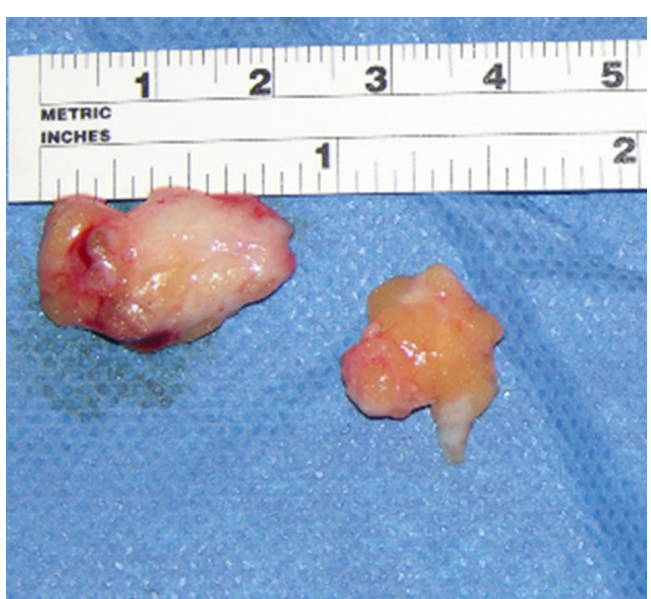

Fig. 3 Gross specimen demonstrating the firm yellow mass without an appreciable stalk or association with any neurologic structure, as well as some surrounding adipose tissue

decreased function, recurrence, or complications 3 months after her procedure.

\section{Discussion}

A schwannoma is a solitary benign proliferation of nerve sheath cells that often presents as a mass along a peripheral nerve. These lesions typically present with normal radiographs and bright signal on T2 MRI in individuals between 20 and 50 years of age with a female predominance [5]. Histologic examination demonstrates biphasic pattern of Antoni type A: organized proliferations of spindle cells and Antoni type B: hypocellular myxoid matrix, regions. Treatment includes observation or meticulous surgical excisional biopsy [13].

A variety of unusual presentations of schwannomas in the hand have been described $[2,8,11,14,16,18,19]$. The rare schwannoma associated with a digital nerve is more commonly found on the palm [6], but lesions distal to the proximal interphalangeal joint have been described $[9,17]$. As well, rare intraosseous schwannomas that arise from within bone without an obvious associated nerve have been described [3, 4]. Literature review, however, did not identify any reports of the unusual presentation of a schwannoma with characteristics analogous to that of a mucous cyst or located at the dorsal aspect of the DIP.

Given their usual presentation as soft, slow growing masses in the upper extremity, schwannomas are frequently misdiagnosed pre-operatively. Ozdemir et al. revealed the difficulty in preoperative diagnoses of schwannomas in the hand and wrist in their literature review and case series. Nine of fourteen patients with schwannomas were misdiagnosed as 
Fig. 4 a and b Photomicrographs demonstrating: the fibrous peripheral encapsulation $(+)$ surrounding spindle cells in a myxoid stroma $(x)$ on low power (a), further detail of the hyperchromatic arranged spindle cells $(+)$ and surrounding hypocellular areas $(x)$ on high power (b)
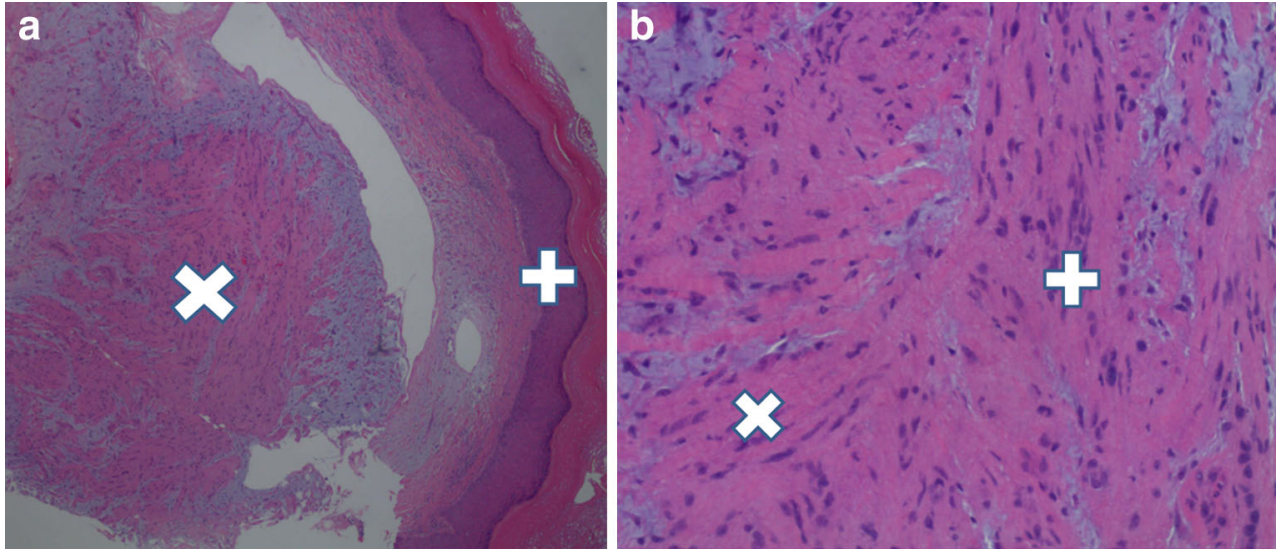

having the more common ganglion, fibroma, xanthoma, or lipoma [12]. Our patient presented with many of the characteristic features of a mucous cyst, having a solitary asymptomatic painless mass about the dorsal aspect of the DIP joint with associated grooving of the nail plate. The patient's radiographs, however, did not demonstrate any obvious osteoarthritis about the DIP joint which is a common feature of mucous cysts, identified in 122 out of a series of 134 patients described by Rizzo [15]. As well as this, our patient's presentation was inconsistent with a typical schwannoma, as the mass was without associated paresthesia and was not located on the flexor surface [7]. Interestingly, upon excision of the mass, no discreet nerve could be identified as the site of origin for the schwannoma, and the patient has never experienced a neurologic deficit. Histological and immunohistochemical investigations performed following surgical excision identified the diagnostic features of a myxoid schwannoma, having organized proliferation of spindle cells in a hypocellular myxoid stroma that demonstrates S-100 immunoreactivity.

This tumor was unusual in that it clinically had the appearance of a mucous cyst; a dorsal mass at the DIP joint with an associated nail ridge. The aim of this report was to identify the unique presentation of a schwannoma in the hand that mimics a mucous cyst. This unusual presentation encourages hand surgeons to maintain a broad, expandable differential diagnosis throughout the work-up and treatment of typical appearing lesions.

\section{Source of funding/disclaimer None.}

Conflict of Interest C. Liam Dwyer, Andrew J. Creager, and John D. Lubahn declare that we have no conflict of interest.

Statement of Human and Animal Rights All procedures followed were in accordance with the ethical standards of the responsible committee on human experimentation (institutional and national) and with the Helsinki Declaration of 1975, as revised in 2008 (5).
"Statement of Informed Consent" Informed consent was obtained from all patients for being included in the study.

\section{References}

1. Adani R, Baccarani A, Guidi E, et al. Schwannomas of the upper extremity: diagnosis and treatment. Chir Organi Mov. 2008;92:85-8.

2. Afshar A, Afaghi F. Intraosseous schwannoma of the second metacarpal: case report. J Hand Surg [Am]. 2010;35:776-9.

3. de la Monte SM, Dorfman HD, Chandra R, et al. Intraosseous schwannoma: histologic features, ultrastructure, and review of the literature. Hum Pathol. 1984;15:551-8.

4. Fawcett KJ, Dahlin DC. Neurilemmoma of bone. Am J Clin Pathol. 1967;47:759-66.

5. Hajdu SI. Schwannomas. Mod Pathol. 1995;8:109-15.

6. Han KJ, Lee YS, Park M. Digital nerve schwannoma of the hand. J Hand Surg Eur Vol. 2012;37:361-2.

7. Holdsworth BJ. Nerve tumours in the upper limb. A clinical review. J Hand Surg (Br). 1985;10:236-8.

8. Huntley JS, Davie RM, Hooper G. A subungual schwannoma. Plast Reconstr Surg. 2006;117:712-3.

9. Kilic A, Cinar C, Arslan H, et al. Re: a benign schwannoma of the digital nerve distal to the proximal interphalangeal joint. J Hand Surg Eur Vol. 2008;33:212-3.

10. Kransdorf MJ. Benign soft-tissue tumors in a large referral population: distribution of specific diagnoses by age, sex, and location. Am J Roentgenol. 1995;164:395-402.

11. Kujala S, Kallioinen M, Forsman M, et al. Intraosseous schwannoma of the middle phalanx. Scand J Plast Reconstr Surg Hand Surg. 2006;40:318-20.

12. Ozdemir O, Ozsoy MH, Kurt C, et al. Schwannomas of the hand and wrist: long-term results and review of the literature. J Orthop Surg. 2005; 13:267-72.

13. Payne WT, Merrell G. Benign bony and soft tissue tumors of the hand. J Hand Surg [Am]. 2010;35:1901-10.

14. Posner MA, McMahon MS, Desai P. Plexiform schwannoma (neurilemmoma) associated with macrodactyly: a case report. J Hand Surg [Am]. 1996;21:707-10.

15. Rizzo M, Beckenbaugh RD. Treatment of mucous cysts of the fingers: review of 134 cases with minimum 2-year follow-up evaluation. J Hand Surg [Am]. 2003;28:519-24. 
16. Robb JE. Trigger finger due to neurilemmoma in the carpal tunnel. Hand. 1978;10:299-301.

17. Rockwell GM, Thoma A, Salama S. Schwannoma of the hand and wrist. Plast Reconstr Surg. 2003;111:1227-32.
18. Ruda R, Kucharzyk DW, Roy DR, et al. Digital schwannoma in a skeletally immature child. J Hand Surg [Am]. 1991;16(2):248-50.

19. Yamamoto T, Fujioka H, Mizuno K. Malignant schwannoma of the digital nerve in a child. A case report. Clin Orthop Relat Res. 2000;376:209-12. 